\title{
SMALL SETS OF EVEN TYPE AND CODEWORDS
}

Dedicated to Professor Helmut Karzel on the occasion of his $70^{\text {th }}$ birthday

J. D. Key and M. J. de Resmini

We examine some geometric configurations of points in designs that give rise to vectors in the codes associated with the designs. In particular we look at small sets of points in projective planes of even order that are met evenly by all the lines of the plane, and find vectors of small weight in the binary hull and in the code's orthogonal.

\section{INTRODUCTION}

Given any design or incidence structure $\mathcal{D}$, for any prime $p$ we define the $p$-ary code of $\mathcal{D}$ to be the vector space $C$ spanned over the field $F_{p}$ of order $p$ by the incidence vectors of the blocks of $\mathcal{D}$. In [1] the code $C$, its orthogonal code $C^{\perp}$, the hull $\left(C \cap C^{\perp}\right)$ of $\mathcal{D}$, and $C+C^{\perp}$, are studied. We look here for the occurrence of the incidence vectors of certain geometric structures in these codes, extending some of the ideas from [4].

Suppose $\mathcal{D}$ is a $2-(v, k, \lambda)$ design, and let $C$ be its code over a field $F$. Evidently the minimum weight of $C$ will be at most the block size, $k$. For the orthogonal code $C^{\perp}$, or the hull $C \cap C^{\perp}$, knowledge of the minimum weight or the minimum-weight vectors might not be easy to deduce. It is well known (see [1, Lemma 2.4.2] for a proof) that if $C$ is not the full space then $C^{\perp}$ has minimum weight at least $(r+\lambda) / \lambda$, where $r$ is the replication number of the design, i.e. the number of blocks through a point, $(v-1) \lambda /(k-1)$. Further, if $F$ has characteristic 2 and $\mathcal{D}$ has even order, then a word of this weight in $C^{\perp}$ will have support that is an oval for $\mathcal{D}$. A natural question arises as to what the minimum weight of $C^{\perp}$ is in the case where $\mathcal{D}$ is known not to have ovals.

Our strategy here will be to look at sets of points of a design that intersect the blocks of the design in a particular way, and thus to deduce that the incidence vector of the set of points 
is in one of the codes mentioned above. We will restrict our attention in this paper mainly to the case of projective planes of even order. Our main results concern the existence of words of weight $2 n$ that are not the difference of the incidence vectors of two lines, in the binary hull of some classes of non-desarguesian projective planes of even order $n$ (see Section 4 , Proposition 2 and Corollary 3), and words of weight $n+4$ in the binary orthogonal code of even-order projective planes (Section 5). In particular we exhibit such sets in all the 22 known planes of order 16 (Section 6) and thus obtain the proposition:

Proposition 1 All the 22 known projective planes of order 16 have words of weight 20 in their orthogonal binary codes, and four of these codes have 20 as the minimum weight.

In Section 6 we also exhibit some new complete 16-arcs for one of the planes of order 16, and also some even sets of sizes other than 20 .

First we review some terminology.

\section{BACKGROUND}

The notation used is generally standard and we refer the reader to Assmus and Key [1].

An incidence structure $\mathcal{D}=(\mathcal{P}, \mathcal{B})$ with point set $\mathcal{P}$ and block set $\mathcal{B}$ is a $t$ - $(v, k, \lambda)$ design if every block is incident with precisely $k$ points and any set of $t$ distinct points are together incident with precisely $\lambda$ blocks. It follows (see [1, Chapter 1]) that $\mathcal{D}$ is an $s$-design for any $s<t$; we denote the number of blocks incident with $s$ points by $\lambda_{s}$. The order of a $t$-design, where $t \geq 2$, is $n=\lambda_{1}-\lambda_{2}$. A Steiner design has $\lambda=1$. A symmetric design is a 2-design with $|\mathcal{P}|=|\mathcal{B}|$, and in this case the dual structure $\mathcal{D}^{t}=(\mathcal{B}, \mathcal{P})$ is also a symmetric design, with the same parameters. A projective plane is a symmetric design with $\lambda=1$.

For $F$ any field, $F^{\mathcal{P}}$ is the vector space of functions from $\mathcal{P}$ to $F$ with basis given by the characteristic functions of the singleton subsets of $\mathcal{P}$. If $\mathcal{D}=(\mathcal{P}, \mathcal{B})$ is an incidence structure, the code $C_{F}(\mathcal{D})$ of $\mathcal{D}$ over $F$ is the subspace of $F^{\mathcal{P}}$ spanned by the characteristic functions (incidence vectors) of the blocks of $\mathcal{D}$. If $X \subseteq \mathcal{P}$, denoting the characteristic function on $X$ by $v^{X}$, then $C_{F}(\mathcal{D})=\left\langle v^{B} \mid B \in \mathcal{B}\right\rangle$. If $F$ has characteristic $p$ (in fact, $F=F_{p}$ ) then the dimension of $C_{F}(\mathcal{D})$ is referred to as the $p$-rank of $\mathcal{D}$. It is well known that the code of a design of order $n$ will only be of any interest or use in characterizations of the design when the prime $p$ divides $n$ : see [1], for example. Since we shall also be looking at the code of the dual structure of $\mathcal{D}$, i.e. $\mathcal{D}^{t}$, we introduce also the notation that was used in [1] for incidence vectors in the space $F^{\mathcal{B}}$, viz. $w^{T}$, where $T \subseteq \mathcal{B}$. Thus $w^{x}$, where $x \in \mathcal{P}$, is the incidence vector of a block in $\mathcal{D}^{t}$, and has weight $r$.

The orthogonal code $C^{\perp}$ (where the orthogonal is taken with respect to the standard inner 
product in $F^{v}$, i.e. , for $\left.u, w \in F^{v},(u, w)=\sum_{x \in \mathcal{P}} u(x) w(x)\right)$ is defined by

$$
C^{\perp}=\left\{u \mid u \in F^{v} \text { and }(u, w)=0 \text { for all } w \in C\right\} .
$$

The hull of a design $\mathcal{D}$ with code $C$ over the field $F$ is the code

$$
\operatorname{Hull}_{F}(\mathcal{D})=H_{F}(\mathcal{D})=C \cap C^{\perp}
$$

Recall that the weight of a vector is the number of non-zero entries in the vector. Clearly the code from a design will have minimum weight at most the block size $k$. The vector in $F^{v}$, all of whose entries are 1 , is called the all-one vector and denoted by $\boldsymbol{J}$. Thus $\boldsymbol{J}=v^{\mathcal{P}}$.

Let $\mathcal{D}=(\mathcal{P}, \mathcal{B})$ be a $2-(v, k, \lambda)$ design. A set of points $\mathcal{S} \subseteq \mathcal{P}$ such that no three points of $\mathcal{S}$ are together on a block of $\mathcal{B}$ is called an arc. A block $B \in \mathcal{B}$ is

- tangent to $\mathcal{S}$ if $B$ meets $\mathcal{S}$ in a single point;

- secant to $\mathcal{S}$ if $B$ meets $\mathcal{S}$ in two points;

- exterior to $\mathcal{S}$ if $B$ does not meet $\mathcal{S}$.

(More generally, if $\mathcal{S}$ is a set of points in $\mathcal{D}$, then a block is called a $j$-secant if it meets $\mathcal{S}$ in $j$ points.) In general, we will use the following notation for the intersections of blocks with a set $\mathcal{S}$ of points in a design:

- $t_{j}$ is the number of $j$-secants to $\mathcal{S}$;

- $u_{j}$ is the number of $j$-secants through a point not on $\mathcal{S}$;

- $v_{j}$ is the number of $j$-secants through a point on $\mathcal{S}$.

Thus $u_{j}$ and $v_{j}$ will depend on the point chosen, in general.

It is easy to show (see $[1$, Chapter 1$])$ that if $\mathcal{D}=(\mathcal{P}, \mathcal{B})$ is a $2-(v, k, \lambda)$ design of order $n$, where $k \geq 3$, and if $S$ is an $s$-arc of $\mathcal{D}$, then

(1) if $n$ is odd, or $n$ is even and $\lambda$ does not divide $r, s \leq(r+\lambda-1) / \lambda$;

(2) if $n$ is even and $\lambda$ divides $r, s \leq(r+\lambda) / \lambda$.

An oval (usually called a hyperoval in the literature when $\mathcal{D}$ is a projective plane of even order) in $\mathcal{D}$ is an arc of maximum size $m$, where

(1) $m=(r+\lambda-1) / \lambda$ if $n$ is odd, or if $n$ is even and $\lambda$ does not divide $r$ (i.e. every point on the arc is on a unique tangent); 
(2) $m=(r+\lambda) / \lambda$ if $n$ is even and $\lambda$ divides $r$ (i.e. there are no tangents).

An $\operatorname{arc} \mathcal{S}$ is complete if no point can be adjoined without $\mathcal{S}$ losing the property of being an arc. The secants (also called 2-secants) to a complete arc cover all the points off the arc. Given a set of points $\mathcal{S}$ in a design $\mathcal{D}$ the intersection numbers associated with $\mathcal{S}$ are the sizes of the intersections of the blocks with $\mathcal{S}$. Thus in a 2-design an arc has intersection numbers from the set $\{0,1,2\}$ and an oval (hyperoval) has intersection numbers 0 and 2 .

\section{Sets of Even Type}

Let $\Pi$ be a finite projective plane of order $n$, i.e. $\Pi$ is a $2-\left(n^{2}+n+1, n+1,1\right)$ design. It is well known that the $p$-ary code $C$ of $\Pi$, where $p \mid n$, has minimum weight $n+1$ and the minimum-weight vectors are the multiples of the incidence vectors of the lines of $\Pi$ : see [1, Chapter 6], for example. Thus the code completely determines the plane. In general, less is known about $C^{\perp}$ and the hull; for the desarguesian plane, the minimum weight of the hull is $2 n$, with the minimum-weight vectors the scalar multiples of the difference of two incidence vectors of lines. It is not known if this is the minimum weight for the hull of any plane, but we do know that for some non-desarguesian planes vectors of weight $2 n$ that are not of this form may be present in the hull: see below. Similarly, not a great deal is known about $C^{\perp}$, except that for binary codes the minimum weight is at least the size of a hyperoval, i.e. $n+2$, and any vector of this weight in $C^{\perp}$ must be the incidence vector of a hyperoval. In the case where $p$ is odd, even the minimum weight of $C^{\perp}$ is not known in general. Until quite recently there was no finite plane known that had no hyperoval; however, four planes of order 16 are now known to have no hyperovals: see [10]. The question then arises: what is the minimum weight of $C^{\perp}$ ? Is it then $n+4$, the next possible weight? We show in Section 6 that in the case of the 22 known planes of order 16 vectors of weight 20 can be constructed in $C^{\perp}$. We also look at the general question: are there always weight- $(n+4)$ vectors in the binary code $C^{\perp}$ when $\mathrm{n}$ is even?

A non-empty set $\mathcal{S}$ of points in a plane is said to be of even type if every line of the plane meets it evenly. It follows that $|\mathcal{S}|$ and the order $n$ of the plane must be even, and that $|\mathcal{S}|=n+2 s$, where $s \geq 1$. The incidence vector of a set of even type is thus clearly in the orthogonal binary code of the plane. More specifically, a set of points will be said to have type $\left(n_{1}, n_{2}, \ldots, n_{k}\right)$ if any line meets it in $n_{i}$ points for some $i$, and for each $i$ there is at least one line that meets it in $n_{i}$ points. Thus the set is of even type if all the $n_{i}$ are even. 


\section{Words OF Weight-2n IN THE HulL}

Consider the Hall plane $\Pi$ of order 16, as described in [15]. This plane has complete 16-arcs. If $\mathcal{A}$ is a complete 16 -arc then in the dual plane $\Pi^{t}$ the 32 tangents to $\mathcal{A}$ form a set of even type. Computationally, using the construction described in [15] and Magma [2], and the first complete 16-arc in [15, Section 6], we obtained a vector of weight 32 in the hull of $\Pi^{t}$ that is not the difference (sum) of the incidence vectors of two lines, since lines meet the set in $0,2,4,6$ or 8 points.

More generally, the Hall plane of order $q^{2}$, when $q=2^{m}$ and $m \geq 2$, has complete $q^{2}$-arcs, by Menichetti [7], and the $2 q^{2}$ tangents to such an arc form a set of even type in the dual plane. This will always give us words of weight $2 n$ in the binary hull of such planes of even order $n$, as the following proposition shows.

Proposition 2 Let $\Pi$ be a projective plane of even order $n$ and suppose that $\mathcal{S}$ is a complete $n$-arc in $\Pi$. Then the set $\mathcal{T}$ of tangents to $\mathcal{S}$ in the dual plane $\Pi^{t}$ has incidence vector $w^{\mathcal{T}}$ in the binary hull of $\Pi^{t}$. Furthermore, the vector $w^{\mathcal{T}}$ is not the difference of the incidence vectors of two lines in $\Pi^{t}$.

Proof: Every point $x \in \mathcal{S}$ is on two tangents, so clearly there are $2 n$ tangents. Let $\mathcal{T}$ denote the set of tangents, as points in the dual plane $\Pi^{t}$. Then $\mathcal{T}$ is a set of even type in $\Pi^{t}$. Thus $w^{\mathcal{T}} \in\left(C^{t}\right)^{\perp}$, where $C^{t}$ is the binary code of $\Pi^{t}$.

Now we show that $w^{\mathcal{T}} \in C^{t}$ and thus is also in $\operatorname{Hull}\left(\Pi^{t}\right)$. For this notice that if $w=\sum_{x \in \mathcal{S}} w^{x}$ then

$$
w(L)= \begin{cases}1 & \text { if } L \text { is a tangent } \\ 0 & \text { if } L \text { is a secant or exterior }\end{cases}
$$

and so $w=w^{\mathcal{T}} \in C^{t}$.

Finally, we need show that $w^{\mathcal{T}}$ is not the difference of the incidence vectors of two lines of $\Pi^{t}$, i.e. we need show that no point of $\Pi$ is on $n$ tangents to $\mathcal{S}$. But this is clear since $\mathcal{S}$ is a complete $n$-arc.

Menichetti's result gives us the following:

Corollary 3 Every dual Hall plane of even square order $n$ has a vector of weight $2 n$ in its binary hull that is not the difference of the incidence vectors of two lines.

There are complete 16-arcs in the dual derived semifield plane of order 16, as we will exhibit in Section 6 (13), and thus the derived semifield plane will also have vectors of weight 32 in its binary hull that are not the difference of the incidence vectors of two lines. Similarly, there are complete 16-arcs in the Johnson-Walker plane, as shown in [14]. 
Notice, however, that we are still not able to settle the question of the actual minimum weight of the hull of a plane. However, Proposition 2 does verify once again the well-known result (see, for example, Hirschfeld [3]) that there are no complete arcs of size $n$ in a desarguesian plane of order $n$, since we know from coding theory that the only words of weight $2 n$ in the binary hull of a desarguesian plane of order $n$ are the differences of the incidence vectors of two lines: see [1, Chapters 5,6]. These codes are generalized Reed-Muller codes.

\section{Sets of Even Type of Size $n+4$}

If $\mathcal{S}$ is a set of even type in an even-order plane then $v^{\mathcal{S}} \in C^{\perp}$. The minimum possible weight of vectors in $C^{\perp}$ is $n+2$, in which case the set is a hyperoval. If no hyperovals are present we would like to know what the minimum weight of $C^{\perp}$ can be, and, in particular, if it is $n+4$. We know it is at most $2 n$, since the difference of the incidence vectors of two lines has this weight. We first make the following observation:

Lemma 4 If a set $\mathcal{S}$ of size $n+4$ in a plane of even order $n$ is of even type, then it is of type $(0,2,4)$.

Proof: Let $x$ be a point of $\mathcal{S}$ and let $v_{j}$ be the number of $j$-secants through it. Counting gives

$$
\begin{aligned}
\sum_{i=1} v_{2 i} & =n+1 \\
\sum_{i=1}(2 i-1) v_{2 i} & =n+3
\end{aligned}
$$

which gives

$$
\sum_{i=1}(2 i-2) v_{2 i}=2
$$

and thus $v_{2 i}=0$ for $i \geq 3, v_{4}=1$, and we have the result.

Migliori [8] considered generally sets of size $n+s$ and of even type $(0,2, s)$, when the order $n$ of the plane is even, and in particular she obtains such sets for $s=\frac{1}{2} n$ in desarguesian planes. This of course gives vectors of weight $n+\frac{1}{2} n$ in $C^{\perp}$.

Korchmáros and Mazzocca [5] consider $(n+t)$-sets of type $(0,2, t)$ in the desarguesian plane of order $n$. They show that sets of size $n+4$ of type $(0,2,4)$ always exist in the desarguesian planes of orders $n=4,8,16$, but have no existence results beyond this for even sets of size $n+4$. With Magma we constructed some of the sets described in that paper in the desarguesian plane of order 16; the set described in their Proposition 7.1 (the statement of which has a printing error in that $\eta^{9}$ should read $\eta^{7}$ ) is the union of two complete 10-arcs 
that are mapped onto one another by an elation of the plane. In fact it seems that a 20 -set of type $(0,2,4)$ can always be split into two complete 10 -arcs by some choice of two points on each of the five 4 -secants. The same appears to hold for 12 -sets of type $(0,2,4)$ in the plane of order 8. (Note again that there is an error in the statement of Theorem 4 of [5]: for $\left(a^{2}+1\right)$ and $\left(a^{2}+a\right)$ read their inverses. Similarly, in Theorem 5 , for the polynomial $f_{2}$ the set of $w_{i}$ should read $\left\{w^{4}, w^{11}, w^{13}\right\}$.)

We will show in Section 6 that all the known planes of order 16 have sets of even type of size 20, and thus $C^{\perp}$ has minimum weight 18 in the case where the plane has hyperovals, or 20 in the case of the four planes that do not have hyperovals.

First we make some general observations about sets of type $(0,2,4)$. If $\mathcal{S}$ is such then, as we observed before, $|\mathcal{S}|=n+2 s$ where $s \geq 1$. Using the notation defined in Section 2 , for any point on $\mathcal{S}$, let $v_{j}$ denote the number of $j$-secants through it. Then $v_{2}+v_{4}=n+1$ and $v_{2}+3 v_{4}=n+2 s-1$, so that $v_{4}=s-1$. If $s=2$ then $v_{4}=1$ and we see that the 4 -secants partition the points of $\mathcal{S}$. Further, keeping $s=2$, i.e. $|\mathcal{S}|=n+4$, if we denote by $t_{j}$ the number of $j$-secants to $\mathcal{S}$, for $j \in\{0,2,4\}$, then counting shows that $t_{4}=\frac{1}{4}(n+4)$, $t_{2}=\frac{1}{2} n(n+4)$ and $t_{0}=\frac{1}{4} n(2 n-5)$. Finally take a point not on $\mathcal{S}$ and let $u_{j}$ denote the number of $j$-secants through it. Then $u_{0}+u_{2}+u_{4}=n+1$ and $2 u_{2}+4 u_{4}=n+4$.

In all but one of the cases we have constructed, i.e. for $n \leq 16$, and also for the desarguesian planes in general (from [5, Theorem 1, page 448]), the 4-secants are concurrent. Following [5] we will refer to such a point as the 4 -nucleus of the 20 -set. This point would have $u_{2}=0$ and $u_{4}=\frac{1}{4} n+1$. Notice that since $u_{2}=\frac{1}{2} n+2-2 u_{4}$ and $n \geq 4, u_{2}$ is always even. It is clear that it is not possible to have a point with $u_{4}=\frac{1}{4} n$, since in that case the last 4 -secant cannot be constructed; thus in particular, for $n=8$, which is the desarguesian case proved already in [5], the 4-secants must be concurrent. The 20 -set without a nucleus that we found by computation in one of the planes of order 16 has two points with $u_{4}=3$ and four with $u_{4}=2$ : see Section 6 (17). The five 4 -secants and the six points of intersection have the property that they can be completed to a Fano plane by the addition of one more point and two more lines.

We consider now possible constructions of $(n+4)$-sets of type $(0,2,4)$. First note that we need $n \geq 4$, and that for $n=4$ we simply take the symmetric difference of two lines. Thus we take $n \geq 8$. A possible construction of an $(n+4)$-set of type $(0,2,4)$ involves the existence of complete $\left(\frac{1}{2} n+2\right)$-arcs:

Proposition 5 Suppose the projective plane $\Pi$ of even order $n$ has two complete $\left(\frac{1}{2} n+2\right)$ $\operatorname{arcs} \mathcal{A}$ and $\mathcal{A}^{\prime}$, with the following properties:

(i) $\mathcal{A}$ and $\mathcal{A}^{\prime}$ share exactly one interior point (i.e. point on no tangent) and the $\frac{1}{4} n+1$ secants on it;

(ii) the $\frac{1}{2} n\left(\frac{1}{2} n+2\right)$ tangents to $\mathcal{A}$ are also tangents to $\mathcal{A}^{\prime}$, and conversely; 
(iii) the $\left(\begin{array}{c}\frac{1}{2} n+2 \\ 2\end{array}\right)-\left(\frac{1}{4} n+1\right)=\frac{1}{8} n^{2}+\frac{1}{2} n$ secants to $\mathcal{A}$ other than the $\frac{1}{4} n+1$ secants on the common interior point are exterior to $\mathcal{A}^{\prime}$, and similarly interchanging $\mathcal{A}$ and $\mathcal{A}^{\prime}$.

Then $\mathcal{A} \cup \mathcal{A}^{\prime}$ is an $(n+4)$-set of type $(0,2,4)$.

Proof: The proof is straightforward.

In fact for the planes of order 16 we have found 20-sets of type $(0,2,4)$ arising from this construction, apart from the one example where no 4-nucleus was present. Conversely, starting with a 20 -set $\mathcal{S}$ of type $(0,2,4)$ with a 4 -nucleus, in all the cases we computed we found that we could choose any subset $\mathcal{T}$ of $\mathcal{S}$ such that $\mathcal{T}$ had one point from one of the 4 -secants, say $L$, but met all the other 4 -secants twice, then a point $x$ could be found on $L \cap \mathcal{S}$ such that $\mathcal{T} \cup\{x\}$ turned out to be a complete 10-arc. This is also true for the planes of order 4 and 8 , and might follow generally. In the one case of a 20-set without a 4 -nucleus the set still split in many ways into two complete 10 -arcs, but the choices were not quite as free. Furthermore, for some of the complete 10-arcs from some splittings in some of the planes, once the finite points are chosen, there may or may not be some freedom in the points at infinity on the 10-arc.

We also note a rather obvious fact that can be employed in the search for $(n+4)$-sets: our primary interest was in the construction of $(n+4)$-sets of even type in the case when no hyperoval is present in the plane, since this would give the minimum weight of the plane's binary code's orthogonal as $n+4$. However, since we are interested now in the existence in all planes of $(n+4)$-sets of even type, we note the following construction that quite frequently can be made:

Proposition 6 If a projective plane $\Pi$ of even order $n$ has two hyperovals that intersect in exactly $\frac{1}{2} n$ points, then the symmetric difference of the two hyperovals is an $(n+4)$-set of even type.

Proof: In the orthogonal binary code the sum of the two incidence vectors of the two intersecting hyperovals has support of weight $n+4$. Since it is clearly a set of even type, it is of type $(0,2,4)$, by Lemma 4 , as required.

Note that the two arcs of size $\frac{1}{2} n+2$ that are the non-intersecting parts of the pair of hyperovals are obviously not two complete $\left(\frac{1}{2} n+2\right)$-arcs that the $(n+4)$-set might split into. Such a splitting must be found from other considerations. Notice also that not all planes have such pairs of intersecting hyperovals, and in particular not all of the planes of order 16 do.

We now give general constructions of 20-sets of type $(0,2,4)$ with a 4 -nucleus in the known planes of order 16 . The existence of these sets with a 4 -nucleus obtained by these constructions in the known planes of order 16 is a consequence of the fact that all of these planes have 
some 4-group acting on them. It may be a Baer 4-group or simply some subgroup of the elementary abelian 2-group acting on the plane. The non-translation planes also admit some translations and/or shears. All these planes have a distinguished line (the line at infinity) and/or a distinguished point; furthermore, in many cases there is a distinguished flag.

Construction 7 Let $\Pi$ be any of the known planes of order 16 . Then $\Pi$ has a 20-set of even type.

Proof: To construct a 20-set we start with two of its 4-secants. Denoting the set of eight starting points by $S$, these points yield sixteen 2 -secants, all of whose points not in $S$ become forbidden points, i.e. they cannot be added to $S$ in order to get a 20-set of even type.

If the sixteen 2-secants come in pencils and these pencils have collinear centres, then we can find points to add to $S$. For this we need $S$ to have a Klein 4-group $V_{4}$ in its stabilizer. Then the two 4-secants which form $S$ must meet on the distinguished line (possibly at the distinguished point).

One way that guarantees such a situation is to choose as $S$ the set of finite points on two lines of a Baer subplane $\Pi_{0}$ of $\Pi$ with the line at infinity of $\Pi_{0}$ coinciding with the line at infinity, $a 0$, of $\Pi$. Let $I$ be the point on $a 0$ where the two 4 -secants meet. Since $\Pi_{0}$ is a Baer subplane of $\Pi$, it is a blocking set. Thus the sixteen 2 -secants produced by $S$ kill all points of $\Pi$ but those of $\Pi-\Pi_{0}$ on the three lines of $\Pi_{0}$ through $I$ other than the two starting 4-secants. This leaves $3 \cdot 12$ points that can be added to $S$ to complete the 20 -set. Indeed there are usually three possible choices and again the eight finite points that are added to $S$ must be chosen so that they are stabilized by the same $V_{4}$ that stabilizes $S$. With such a choice the four points at infinity that the 20-set has come out naturally. (If $\Pi$ is a shear plane then $a 0$ is substituted by a shear axis.)

Moreover, to construct a 20 -set with 4 -nucleus it is enough to choose the set $S$ of eight starting points on two 4-secants so that the two 4-secants meet at a point $I$ on the distinguished line of $\Pi$ (possibly $I=A 0$ where $A 0$ is the distinguished point of $\Pi$ ) and $S$ is stabilized by a $V_{4}$. Then the sixteen 2 -secants provided by $S$ come in pencils with centres on the distinguished line of $\Pi$ (on a shear axis). Once the forbidden points are deleted, points remain on lines through $I$ that can be added to $S$, using the $V_{4}$ to pick them out. Thus a 20 -set is constructed. Usually we get six 20-sets starting from $S$.

\section{Some 20-Sets in the Known Planes of Order 16}

We give in this section specific 20-sets of even type for the known planes of order 16 . In most of the cases we are able to describe the specific sets with reference to published papers 
constructing the plane, but in some cases we had to resort to pure computation, using Magma, looking for words of weight 20 in the code's orthogonal, and then analysing the structure of the set. In such cases we refer the interested reader to the first author for data containing the plane and the 20-set.

All the planes have 20-sets; we refer to the notation used in the various papers, as quoted. All the sets found by construction have a 4-nucleus, and all split into two complete 10-arcs, in many ways. We indicate a splitting in each case, giving just one of the complementary complete 10-arcs. Note that the desarguesian plane of order 16 has these sets and they can be constructed as described earlier, using the results of [5]. The existence of the 20-sets that we describe below is in all cases a consequence of the existence of an elementary abelian 2-group acting on the plane. In the notation for these planes, the points labelled $A i$ are the points on the line at infinity, labelled $a 0$. The remaining points are the finite points.

The method of obtaining the 20-sets is either that outlined in Proposition 6 or Construction 7 . In the latter case, starting with eight points, if the eight points are together in a Baer subplane then three distinct 20-sets arise; if the eight points are not in a common Baer subplane then more 20-sets might arise. Similarly, it will be noticed that in certain situations for some of the planes the complementary complete 10 -arcs have the property that their finite points might be completed to two hyperovals whose intersection is the set of finite points. This then produces yet another 20-set. Furthermore, different splittings into complete 10-arcs might yield distinct possibilities.

The planes are enumerated by ascending 2-rank, which is noted in each case, along with the symbols used to denote the designs as in [10]. To be consistent with that notation we have denoted the dual of a plane $\Pi$ by $\Pi^{*}$. We have included 20 -sets for the planes that we examined computationally; in this case the plane is obtained from the ftp site cs.uwa.edu.au in the directory pub/graphs/planes16 [9].

Remark 1 The points in our case are labelled 1 to 273 instead of 0 to 272 . Thus to retrieve the plane as given in the ftp site [9], subtract 1 from each point in our notation.

We can omit the desarguesian plane, since 20-sets can be constructed from [5], but since we found two hyperovals (one regular and one Lunelli-Sce [6]) that intersect in eight points, we will include these two hyperovals, in terms of homogeneous coordinates, just for completeness.

The sets given below then constitute a proof of Proposition 1 as stated in Section 1.

(1) Desarguesian plane $\left(\mathbf{P G}_{\mathbf{2}} \mathbf{( 1 6 )}\right.$ 2-rank 82)

If $w$ is a root of the primitive polynomial $x^{4}+x+1$ over $F_{2}$, then

$\left(1, w^{14}, w\right),\left(1, w^{12}, w^{3}\right), \quad\left(1, w^{5}, w^{10}\right), \quad\left(1, w^{2}, w^{13}\right),\left(1, w^{8}, w^{7}\right), \quad\left(1, w^{7}, w^{8}\right), \quad\left(1, w^{6}, w^{9}\right)$, $\left(1, w^{9}, w^{6}\right),\left(1, w^{4}, w^{11}\right),(1,0,0),\left(1, w, w^{14}\right),\left(1, w^{11}, w^{4}\right),(0,1,0),\left(1, w^{10}, w^{5}\right),(0,0,1)$, $\left(1, w^{3}, w^{12}\right),\left(1, w^{13}, w^{2}\right),(1,1,1)$ 
is a regular hyperoval and

$\left(1, w^{14}, w\right),\left(1, w^{12}, w^{3}\right), \quad\left(1, w^{5}, w^{10}\right), \quad\left(1, w^{2}, w^{13}\right), \quad\left(1, w^{8}, w^{7}\right), \quad\left(1, w^{7}, w^{8}\right), \quad\left(1, w^{6}, w^{9}\right)$, $\left(1, w^{9}, w^{6}\right), \quad\left(1, w^{12}, w^{9}\right), \quad\left(1, w^{6}, w^{7}\right), \quad\left(1, w^{2}, w^{8}\right), \quad\left(1, w^{5}, w^{4}\right), \quad\left(1, w^{14}, w^{4}\right), \quad\left(1, w^{7}, w\right)$, $\left(1, w^{8}, w^{10}\right),\left(1, w^{9}, w^{3}\right),\left(1, w^{10}, w^{13}\right)\left(1, w^{10}, w^{6}\right)$

is a Lunelli-Sce hyperoval, and they meet in eight points. The resulting 20-set has 4-nucleus $\left(1, w^{10}, w^{4}\right)$ and splits into two complete 10-arcs, one of which is:

$\left(1, w^{10}, w^{5}\right),\left(1, w^{14}, w^{4}\right),(1,0,0),\left(1, w^{11}, w^{4}\right),\left(1, w^{9}, w^{3}\right),(1,1,1) .\left(1, w^{2}, w^{8}\right),(0,0,1)$, $\left(1, w^{6}, w^{7}\right),\left(1, w^{13}, w^{2}\right)$.

(2) Semifield plane with kern $F_{2}$ (SEMI2 2-rank 98) [11]

A pair of hyperovals ([11, page 145], $\gamma 1, \gamma 2$ ) intersecting in eight points gives the 20 -set with 4-nucleus $A 3$ in this self-dual plane:

K13 K14 R13 R14 F5 F16 X5 X16 A15 A16 D8 D15 N8 N15 M9 M11 P9 P11 A4 A14, and a complete 10-arc:

\section{K13 K14 R13 R14 M9 M11 P9 P11 A4 A14.}

Note in addition that the eight finite points of each of the complementary complete 10 -arcs completes uniquely to a hyperoval which for the 10 -arc given above is

K13 K14 R13 R14 M9 M11 P9 P11 B3 B6 L7 L12 W7 W12 Z3 Z6 A2 A13.

Alternatively, starting with the eight points F3P3L3S3F6 P6 L6S6 not in a Baer subplane and using the method of Construction 7, six 20-sets are obtained, all with 4-nucleus $A 1$ :

F3 P3 L3 S3 F6 P6 L6 S6 B9 B11 C9 C11 D9 D11 K9 K11 A4 A7 A14 A16,

F3 P3 L3 S3 F6 P6 L6 S6 B13 B14 C13 C14 D13 D14 K13 K14 A4 A8 A9 A12,

F3 P3 L3 S3 F6 P6 L6 S6 H1 H2 Z1 Z2 N1 N2 R1 R2 A3 A8 A13 A14,

F3 P3 L3 S3 F6 P6 L6 S6 H9 H11 Z9 Z11 N9 N11 R9 R11 A6 A10 A12 A13,

F3 P3 L3 S3 F6 P6 L6 S6 M1 M2 X1 X2 T1 T2 W1W2 A6 A9 A11 A16,

F3 P3 L3 S3 F6 P6 L6 S6 M13 M14 X13 X14 T13 T14 W13 W14 A3 A7 A10 A11.

A splitting of the first of these into complete 10-arcs gives:

B9 B11 C9 C11 F3 F6 P3 P6 A4 A7,

and the eight finite points complete uniquely to a hyperoval:

B9 B11 C9 C11 F3 F6 P3 P6 H4 H10 Z4 Z10 M8 M15 X8 X15 A3 A7.

The point $A 7$ is on the 20 -set, the 10 -arc, and the hyperoval. 
(3) Semifield plane with kern $F_{4}$ (SEMI4 2-rank 98) [17]

This plane also is self-dual. Starting with the points $B 1 B 2 B 13 B 14 C 1 C 2 C 13 C 14$ in the Baer subplane $\alpha_{1}$, using the method of Construction 7, three 20-sets with the 4-nucleus $A 0$ are obtained:

B1 B2 B13 B14C1 C2 C13 C14 T3 T6 W3 W6 T8 T15 W8 W15 A8 A10 A12 A15, B1 B2 B13 B14C1 C2 C13 C14 T4 T10 W4W10 T9 T11 W9 W11 A5 A6 A13 A14, and

B1 B2 B13 B14C1C2C13 C14 T5 T16 T7 T12 W5W16W7W12 A7 A9 A11 A16.

A splitting of the first of these into two complete 10-arcs yields:

B1 B2C1C2T3T6W3W6 A8 A12.

The eight finite points of this 10-arc can be completed to the following two hyperovals that intersect in these eight points and whose symmetric difference gives a 20-set with 4-nucleus $A 0$ :

$$
\text { B1 B2 C1 C2 T3 T6W3W6 A8 A10 D4 D10 K4 K10 M7 M12 X7 X12, }
$$

B1 B2 C1 C2 T3 T6 W3 W6 A12 A15 D9 D11 K9 K11 M5 M16 X5 X16.

Similarly, starting with the points $B 1 D 1 M 1 T 1 F 7 H 7 L 7 R 7$ in the Baer subplane $\beta_{3}$, three 20-sets are obtained, with 4-nucleus $A 1$, one of which is

B1 D1 M1 T1F7 H7 L7 R7 C9 K9 P15 S15 W9 X9 N15 Z15 A0 A8 A13 A16, giving a complete 10-arc:

B1 M1L7R7C9 K9 N15Z15A0A8.

Again, starting with the points $R 1 R 3 R 8 R 13 W 2 W 3 W 11 W 16$ in the Baer subplane $\gamma_{3}$, three 20 -sets are obtained with no points on the line $a 0$ at infinity, with 4-nucleus $A 0$, one of which is

$R 1 R 3 R 8 R 13 W 2 W 3 W 11 W 16$ B1 B2 B4 B10 D6 D8 D10 D11 F4 F6 F13 F16, giving a complete 10-arc:

B1 B2 F4 F6 D6 D8W2W3R1R3. 
Alternatively, one can start with the eight points $B 1 B 2 B 3 B 6 C 1 C 2 C 3 C 6$ not in a Baer subplane and obtain in a similar manner six 20-sets with 4-nucleus $A 0$ :

B1 B2 B3 B6 C1 C2 C3 C6 D4 D10 D7 D12 K4 K10 K 7 K12 A5 A6 A13 A14, B1 B2 B3 B6 C1 C2 C3 C6 D5 D16 D9 D11 K5 K16 K9 K11 A7 A9 A11 A16, B1 B2 B3 B6 C1 C2 C3 C6 L5 L16 L9 L11 S5 S16 S9 S11 A3 A9 A12 A14, B1 B2 B3 B6 C1 C2 C3 C6 L8 L15 L13 L14 S8 S15 S13 S14 A4 A10 A13 A16, B1 B2 B3 B6 C1 C2 C3 C6 N4 N10 N7 N12 R4 R10 R7 R12 A4 A6 A7 A12, B1 B2 B3 B6 C1 C2 C3 C6 N8 N15 N13 N14 R8 R15 R13 R14 A3 A5 A10 A11. A splitting of the first of these to give a complete 10-arc is:

$B 1 B 2 C 1 C 2 D 4 D 10 K 4 K 10 A 5 A 6$.

The eight finite points here again complete to two hyperovals: B1 B2 C1 C2 D4 D10 K4 K10 F8 F15 P8 P15 L5 L16 S5 S16 A6 A15 and B1 B2 C1 C2 D4 D10 K4 K10 M7 M12 X7 X12 T3 T6 W3 W6 A8 A10.

(4) Hall plane (HALL 2-rank 98) [15]

Starting with the eight points $B 1 B 2 B 4 B 10 C 1 C 2 C 4 C 10$ in a Baer subplane, and using Construction 7 , we get three 20-sets with 4-nucleus $A 0$, one of which is:

B1 B2 B4 B10 C1 C2 C4 C10 F9 F11 F13 F14 P9 P11 P13 P14 A9 A11 A13 A14, and a complete 10-arc:

B1 B2 C1 C2 F9 F11 P9 P11 A9 A11.

(In each of the three 20-sets obtained here the eight added finite points are also in a Baer subplane.)

The eight finite points of the complete 10-arc complete to two hyperovals that meet in these eight points:

B1 B2 C1 C2 F9 F11 P9 P11 N3 N6 R3 R6 T5 T16 W5 W16 A9 A13, B1 B2 C1 C2 F9 F11 P9 P11 N7 N12 R7 R12 T8 T15 W8 W15 A11 A14, 
and whose symmetric difference thus gives another 20-set with 4-nucleus $A 0$. (Note that the eight points N3N6R3R6N7N12R7R12 of this 20-set are together on a Baer subplane.)

Alternatively, starting with eight points $B 1 B 2 B 3 B 6 C 1 C 2 C 3 C 6$ not in a Baer subplane, we get five 20 -sets with 4 -nucleus $A 0$, one of which is:

B1 B2 B3 B6 C1 C2 C3 C6 D4 D10 D7 D12 K4 K10 K7 K12 A4 A9 A10 A14,

and a complete 10-arc:

B1 B3 C1C6 D10 D12 K4 K7 A4 A10.

(5) Dual Hall plane (HALL* 2-rank 98)

Using [9] (but see Remark 1) 20-sets with 4-nucleus and splitting into complete 10-arcs were found by computation. A 20-set with 4-nucleus 273 :

$\{1,3,10,12,152,155,158,160,230,231,233,237,242,244,245,255,257,258,265,267\}$,

and a complete 10-arc:

$\{1,12,155,158,231,233,242,255,265,267\}$.

(6) Johnson-Walker plane (JOWK 2-rank 100) [14]

This is one of the planes with pairs of hyperovals meeting in eight points, and thus we can get a 20-set with 4-nucleus $A 0$ from the first pair of hyperovals in Ex. 2, page 136 of $[14]$ :

A7 A12 D3 D6 H3 H6 K 7 K12 Z7 Z12 A8 A15 D5 D16 H5 H16 K8 K15 Z8 Z15, and a complete 10-arc:

A7 A12 D3 D6 H3 H6 K7 K12 Z7 Z8.

Alternatively, starting with eight points $B 1 B 2 B 3 B 6 C 1 C 2 C 3 C 6$ from the Baer subplane $\alpha_{2}$ on page 126 of [14], and using Construction 7, we get three 20-sets with 4-nucleus $A 0$ :

B1 B2 B3 B6 C1 C2 C3 C6 D4 D10 D7 D12 K4 K10 K7 K12 A4 A10 A7 A12, B1 B2 B3 B6 C1 C2 C3 C6 D5 D16 D9 D11 K5 K16 K9 K11 A5 A16 A9 A11, B1 B2 B3 B6 C1 C2 C3 C6 D8 D15 D13 D14 K8 K15 K13 K14 A8 A15 A13 A14. Splitting of the first of these gives a complete 10-arc:

$B 1 B 2 C 1 C 2 D 4 D 10 K 4 K 10 A 4 A 10$. 
(7) Dual Johnson-Walker plane (JOWK* 2-rank 100)

Using [9] (but see Remark 1), 20-sets with 4-nucleus and splitting into complete 10-arcs were found by computation. A 20-set with nucleus 273:

$\{4,8,12,14,215,218,219,224,231,232,234,238,244,251,252,256,258,263,267,271\}$,

and a complete 10-arc:

$\{12,14,215,224,231,232,244,256,258,267\}$.

(8) Dempwolff plane (DEMP 2-rank 102) [12]

Starting with the eight points $B 1 B 2 B 3 B 6 C 1 C 2 C 3 C 6$, which are not in a Baer subplane, and using Construction 7 , we obtain five 20 -sets with 4 -nucleus $A 0$, one of which is:

B1 B2 B3 B6 C1 C2 C3 C6 D4 D10 D7 D12 K4 K10 K7 K12 A3 A4 A8 A14,

containing a complete 10-arc:

$B 1 B 2 C 1 C 2 D 4 D 10 K 4 K 10 A 3 A 4$.

The 2-secants of the 20-set through the original eight points are concurrent in fours at the points $A 1, A 2, A 9, A 15$.

Alternatively, starting with the eight points $B 1 B 2 B 4 B 10 C 1 C 2 C 4 C 10$, which are in the Baer subplane $\alpha$ ([12, page 56]), we obtain three 20 -sets with 4-nucleus $A 0$, one of which is:

B1 B2 B4 B10 C1 C2 C4 C10 F3 F6 F7 F12 P3 P6 P7 P12 A9 A10 A15 A16,

containing a complete 10-arc:

B1 B2 C1C2 F3 F6 P3 P6 A9 A15.

Sets can also be constructed from hyperovals using Proposition 6. For example, using the two hyperovals on page 62 of [12], we get the 20-set with 4-nucleus $A 1$ :

H7 H12 Z7 Z12 N3 N6 R3 R6 A15 A16 M7 M12 X7 X12T3T6 W3 W6 A9 A10, and a complete 10-arc:

H7 H12 Z7 Z12 N3 N6T3T6 A9 A15. 
(9) Dual Dempwolff plane (DEMP* 2-rank 102)

Using [9] (but see Remark 1), 20-sets with 4-nucleus and splitting into complete 10-arcs were found by computation. A 20-set with nucleus 273 :

$\{2,7,8,9,214,219,220,221,231,233,235,236,242,246,248,253,262,265,268,270\}$,

and a complete 10-arc:

$$
\{2,8,220,221,231,233,242,253,265,268\} \text {. }
$$

(10) Lorimer plane (LHMR 2-rank 106) [20]

Pairs of hyperovals intersecting in eight points exist in this plane: the first two on page 147 of [20] give the 20 -set

$$
\text { n15 n16r13r14t15t16x13x14a13a14 p7 p8 s5 s6 w7 w8 z5 z6a11 a12, }
$$

and a complete 10 -arc

$$
n 15 n 16 t 15 t 16 p 7 p 8 w 7 w 8 a 11 a 12 \text {. }
$$

Alternatively, starting with the eight points $d 1 d 2 d 3 d 4 k 1 k 2 k 3 k 4$ not in a Baer subplane, Construction 7 yields six 20-sets with 4 -nucleus $a 0$, one of which is:

$d 1 d 2 d 3 d 4 k 1 k 2 k 3 k 4 r 13 r 14 r 15 r 16 w 13 w 14 w 15$ w16 a3 a5 a9 a15,

and a complete 10 -arc

$$
d 3 d 4 k 3 k 4 r 13 r 14 w 13 w 14 a 3 a 5 .
$$

The eight finite points of this complete 10-arc form the intersection of two hyperovals:

$$
\text { d3 d4 k3 k4r13r14w13w14b11 b12 m11 m12 n5 n6 z5 z6a11a12, }
$$

and

$$
d 3 d 4 k 3 k 4 r 13 r 14 w 13 w 14 c 15 c 16 \ell 15 \ell 16 p 1 \text { p2 x1 x2 a3a4, }
$$

whose symmetric difference is thus a 20 -set.

(11) Dual Lorimer plane (LHMR* 2-rank 106) [20]

Starting with the eight points $B 1 B 2 B 3 B 4 C 1 C 2 C 3 C 4$, which are in a Baer subplane, and using Construction 7 , we obtain three 20 -sets with 4 -nucleus $A 0$, one of which is:

B1 B2 B3 B4C1C2C3C4 D5 D6 D7 D8 F5 F6 F7 F8 A5 A6 A7 A8, containing a complete 10-arc:

B1 B2 C1 C2 D5 D6 F5 F6 A5A6. 
A pair of hyperovals $([20$, page 145$], \omega 1, \omega 2)$ intersecting in eight points gives the 20 -set: N7 N8 R5 R6 T7 T8 X5 X6 A11 A12 P15 P16 S13 S14 W15 W16 Z13 Z14 A13 A14, and a complete 10-arc:

\section{N7 N8T7 T8 P15 P16W15W16 A11 A12.}

Alternatively, starting with the eight points $K 1 K 2 K 3 K 4 P 1 P 2 P 3 P 4$, which are not in a Baer subplane, we obtain six 20 -sets with 4 -nucleus $A 0$, one of which is:

K1 K2 K3 K4 P1 P2 P3 P4 D9 D10 D11 D12 X9 X10 X11 X12 A3 A8 A11 A16, containing a complete 10-arc:

\section{K1 K2 P1 P2 D9 D10 X9X10A3 A8.}

The eight finite points of this arc complete to two hyperovals:

K1 K2 P1 P2 D9 D10 X9 X10 B7 B8 T7 T8 M15 M16 S15 S16 A9 A10

and

K1 K2 P1 P2 D9 D10 X9 X10 C5 C6 W5 W6 L13 L14 R13 R14 A15 A16, whose symmetric difference will give another 20 -set.

We also obtained a 32-set of type $(0,2,4)$ :

B9 B10 B13 B14 C9 C10 C13 C14 X9 X10 X13 X14 Z9 Z10 Z13 Z14

$$
\text { D11 D12 D15 D16 F11 F12 F15 F16 T11 T12 T15 T16 W11 W12 W15 W16. }
$$

It has 56 4-secants which can be partitioned into seven pencils with centres at $A 0, A 1, A 2, A 4, A 5, A 10, A 15$; the remaining lines on each of these points are external. All lines but $a 0$ through any other point on $a 0$ are 2-secants, and there are 160 2-secants.

Furthermore, we obtained a 32-set of type $(0,2,4,8)$ :

$$
\begin{aligned}
& B 1 \text { B2 C1 C2 D5 D6 F5 F6 H7 H8 K7 K8 L3 L4 M3 M4 } \\
& \text { N1 N2 P1 P2 R5 R6 S5 S6 T7 T8W W W8 X3 X4 Z3Z4. }
\end{aligned}
$$

This set has four concurrent 8 -secants, $n 1, n 2, n 9, n 10$, and 324 -secants. 
(12) Derived semifield plane (DSFP 2-rank 106) [13]

A pair of hyperovals ([13, page 108], the first two hyperovals) intersecting in eight points gives the 20-set with 4-nucleus $A 6$ :

H9 H11 T1 T2 W3 W6 Z5 Z16 A8 A12 R9 R11 M1 M2 N5 N16 X3 X6 A7 A13,

and a complete 10-arc:

H9 H11 R9 R11 M1 M2T1T2 A7 A12.

As for some of the other planes already mentioned, the eight finite points of the complete 10-arc complete to two hyperovals whose intersection is this set of eight points:

H9 H11 R9 R11 M1 M2 T1T2 N4 N10 Z4 Z10W13 W14 X13 X14 A3 A7

and

H9 H11 R9 R11 M1 M2 T1 T2 B13 B14 D13 D14 F4 F10 L4 L10 A11 A14,

whose symmetric difference will thus give another 20 -set with 4 -nucleus $A 1$.

Alternatively, starting with points $B 1 B 2 B 5 B 16 C 1 C 2 C 5 C 16$ from a Baer subplane, and using Construction 7 , we obtain three 20 -sets with 4 -nucleus $A 0$, one of which is:

B1 B2 B5 B16 C1 C2 C5 C16 H3 H6 Z3 Z6 H9 H11 Z9 Z11 A3 A6 A9 A11,

and a complete 10-arc:

B1 B2C1C2H3H6Z3Z6A3A6,

and the eight finite points of this arc do not complete to a hyperoval.

(13) Dual derived semifield plane (DSFP* 2-rank 106) [13]

Note that this is one of the planes that does not contain any hyperovals and thus the minimum weight of the plane's binary orthogonal code will be 20 .

Starting with the points $d 1 d 2 d 4 d 10 k 1 k 2 k 4 k 10$ in a Baer subplane $\beta$, and using Construction 7 , three 20 -sets with 4 -nucleus $a 0$ are obtained, one of which is:

$d 1 d 2 d 4 d 10 k 1 k 2 k 4 k 10 \ell 3 \ell 6 s 3 s 6 \ell 7 \ell 12 s 7 s 12 a 3 a 6 a 7 a 12$,

and a complete 10-arc:

$d 1 d 2 k 1 k 2 \ell 3 \ell 6 s 3 s 6 a 3 a 6$.

The finite points of this complete 10-arc and of its complementary arc form parts of four complete 16-arcs:

$d 1 d 2 k 1 k 2 \ell 3 \ell 6 s 3 s 6 h 13 h 14 m 7 m 12 x 9 x 11 z 7 z 12$, 


$$
\begin{gathered}
d 1 d 2 k 1 k 2 \ell 3 \ell 6 s 3 s 6 n 8 n 15 r 7 r 12 t 7 t 12 w 5 w 16, \\
d 4 d 10 k 4 k 10 \ell 7 \ell 12 s 7 s 12 h 9 h 11 m 3 m 6 x 13 x 14 z 3 z 6, \\
d 4 d 10 k 4 k 10 \ell 7 \ell 12 s 7 s 12 n 5 n 16 r 3 r 6 t 3 t 6 w 8 w 15 .
\end{gathered}
$$

(See the comment after Corollary 3 relating to these arcs.)

However, using the starting points $f 3 f 6 f 7 f 12 p 3 p 6 p 7 p 12$ from a Baer subplane and proceeding as before yields again three 20 -sets with 4 -nucleus $a 0$, one of which is:

$$
\text { f3 f6 f7 f12 p3 p6 p7 p12b1 b2 c1 c2 b4b10c4 c10a3a6a7a12, }
$$

with a complete 10-arc

$$
b 1 b 2 c 1 c 2 f 3 f 6 p 3 p 6 a 3 a 6,
$$

but which does not complete to a complete 16-arc on removing the infinite points.

Starting with the eight points $\ell 1 \ell 2 \ell 4 \ell 10 s 1 s 2 s 4 s 10$ in the Baer subplane $\beta$ (see the first example above), yields three 20-sets with 4-nucleus $a 0$, one of which is

$$
\ell 1 \ell 2 \ell 4 \ell 10 s 1 s 2 s 4 s 10 d 3 d 6 d 7 d 12 k 3 k 6 k 7 k 12 a 3 a 6 a 7 a 12
$$

with a complete 10 -arc:

$$
\ell 1 \ell 2 s 1 s 2 d 7 d 12 k 7 k 12 a 3 a 6,
$$

which gives complete 16-arcs from its finite points:

$$
\begin{aligned}
& \ell 1 \ell 2 s 1 s 2 d 7 d 12 k 7 k 12 h 3 h 6 m 13 m 14 x 3 x 6 \text { z9 z11, } \\
& \ell 1 \ell 2 s 1 s 2 d 7 d 12 k 7 k 12 n 3 n 6 r 5 \text { r } 16 t 8 t 15 w 3 w 6 .
\end{aligned}
$$

The symmetric difference of these two complete 16-arcs is not a complete 16-arc.

(14) Mathon plane (MATH 2-rank 109)

Using [9] (but see Remark 1), we found two hyperovals meeting in eight points:

$\{45,46,75,80,99,106,115,126,136,141,152,153,171,176,201,202,264,271\}$,

and

$$
\{1,17,45,62,75,96,106,126,141,153,176,187,202,217,230,246,257,266\},
$$

whose symmetric difference is the 20 -set

$$
\{1,17,46,62,80,96,99,115,136,152,171,187,201,217,230,246,257,264,266,271\}
$$

with 4-nucleus 268, and complete 10-arc

$$
\{1,46,80,99,115,136,171,187,257,264\} .
$$


There are also 20-sets that are not the symmetric difference of two hyperovals:

$\{3,5,11,13,18,24,26,32,227,229,235,237,242,248,250,256,259,260,262,268\}$,

with 4-nucleus 257 and complete 10-arc

$$
\{3,11,24,26,235,237,242,256,259,268\} \text {. }
$$

(15) Dual Mathon plane (MATH* 2-rank 109) [19]

Note that this plane is referred to as the Mathon plane in [19]. Starting with the eight points $B 1 B 2 B 3 B 4 C 1 C 2 C 3 C 4$ in the Baer subplane 17(i) of [19], a 20-set with 4-nucleus $A 0$ is obtained:

B1 B2 B3 B4C1C2C3C4 D5 D6 D7 D8 F5 F6 F7 F8 A5 A6 A7 A8,

and a complete 10-arc:

$B 1 B 2 C 1 C 2 D 5 D 6 F 5 F 6 A 6 A 8$.

(16) Classical semi-translation plane (BBH1 2-rank 110) [18]

This is a self-dual plane. Starting with the points $B 1 B 2 B 3 B 4 C 1 C 2 C 3 C 4$ in a Baer subplane, using the method of Construction 7, three 20-sets with 4-nucleus $A 0$ are obtained, one of which is:

B1 B2 B3 B4 C1 C2 C3 C4 D5 D10 F5 F10 D7 D13 F7 F13 A5 A6 A7 A8,

and a complete 10-arc:

$B 1 B 2 C 1 C 2 D 5 D 10 F 5 F 10 A 5 A 6$.

The eight finite points of this complete 10-arc can be completed to complete 14-arcs in four ways, for example:

$B 1 B 2 C 1 C 2$ D5 D10 F5 F10 H6 H16 P6 P16 A5 A7

and

B1 B2 C1 C2 D5 D10 F5 F10 H12 H15 P12 P15 A6 A8.

New 20-sets with 4-nucleus $A 0$ may be obtained from eight points in the symmetric difference of these two arcs, one of which is:

H6 H16 H12 H15 P6 P16 P12 P15 L1 L2 L3 L4T1 T2 T3 T4 A9 A10 A11 A12.

A 20-set with 4-nucleus $A 0$ with, unusually, no points at infinity was found:

H2 H7 H11 H12 P1 P6 P7 P8 D1 D2 D3 D4 L4 L6 L11 L13 T3 T8 T12 T13, and a complete 10-arc:

H7 H11 P7 P8 D1 D2 L4 L6 T8 T13. 
(17) Derived dual Hall plane (BBH2 2-rank 114)

Using [9] (but see Remark 1), 20-sets were found by computation and some of the sets did not have a 4-nucleus. They all split into two complete 10-arcs. A 20-set without 4-nucleus:

$\{2,3,4,5,7,12,13,16,21,22,28,29,209,210,215,216,217,218,223,224\}$,

and a complete 10-arc:

$\{2,3,12,13,22,28,209,210,223,224\}$.

The five 4-secants of the 20-set meet in a set of six points, and this set can be completed, by the addition of one more point and two more lines, to a Fano subplane. The points of this Fano subplane are

$$
\{17,163,165,167,176,257,265\} \text {. }
$$

A 20-set with 4-nucleus 263:

$\{18,20,25,26,30,32,225,226,227,232,234,237,239,240,259,261,267,269,272,273\}$, and a complete 10-arc:

$\{18,25,26,226,227,232,234,267,269,272\}$.

Neither of these two 20-sets is the symmetric difference of two hyperovals intersecting in eight points, but such hyperovals do exist in this plane:

$\{18,29,82,86,145,154,164,169,177,182,192,200,203,228,238,250,251,264\}$

and

$\{18,19,86,88,93,130,144,145,154,167,169,177,181,185,243,247,250,251\}$, giving the 20-set with 4-nucleus 172:

$\{19,29,82,88,93,130,144,164,167,181,182,185,192,200,203,228,238,243,247,264\}$, and a complete 10-arc:

$\{88,93,144,167,182,200,238,243,247,264\}$.

(18) Dual derived dual Hall plane (BBH2* 2-rank 114)

This is one of the planes without hyperovals. Using [9] (but see Remark 1), 20-sets 
with 4-nucleus and splitting into complete 10-arcs were found by computation. A 20-set with 4-nucleus 9:

$\{14,15,16,32,48,64,80,96,112,128,144,160,176,192,208,224,240,256,272,273\}$,

and a complete 10-arc:

$$
\{14,15,48,80,112,144,176,192,224,256\} \text {. }
$$

(19) Johnson strict semi-translation plane (JOHN 2-rank 114) [16]

Starting with the eight points D3 D6 D4 D10 K3 K6 K4K10 in the Baer subplane at the bottom of page 148 of [16], and using Construction 7, a 20-set with 4-nucleus $A 0$ is obtained:

D3 D6 D4 D10 K3 K6 K4 K10 F5 F16 F13 F14 P5 P16 P13 P14 A3 A6 A4 A10, and a complete 10-arc:

D3 D6 K4 K10 F5 F16 P13 P14 A3 A6.

A different splitting gives the complete 10-arc

D3 D6 K3 K6 F5 F16 P5 P16 A3 A4,

and its complement, to which the following hyperovals are attached:

D3 D6 K3 K6 F5 F16 H13 H14 Z13 Z14 T4T10W4W10 A7 A12,

D3 D6 K3 K6 F13 F14 P13 P14 H4 H10 Z4 Z10 T5 T16 W5 W16 A7 A12.

This plane has only a small number of hyperovals, and no pairs intersecting in eight points.

(20) Dual Johnson strict semi-translation plane (JOHN* 2-rank 114) [16]

This is one of the planes that does not contain any hyperovals and thus the minimum weight of the plane's binary orthogonal code will be 20. Starting with the eight points $d 3 d 6 d 4 d 10 k 3 k 6 k 4 k 10$ in a Baer subplane, and using Construction 7, three 20-sets with 4-nucleus $a 0$ are obtained:

$$
\begin{aligned}
& d 3 d 6 d 4 d 10 k 3 k 6 k 4 k 10 \text { f } 8 \text { f } 15 \text { f9 f } 11 \text { p8 p15 p9 p11 a5 a16 a13 a14, } \\
& d 3 d 6 d 4 d 10 k 3 k 6 k 4 k 10 \text { f5 f } 16 \text { f } 13 \text { f } 14 \text { p } 5 \text { p } 16 \text { p } 13 \text { p } 14 \text { a } 8 \text { a } 15 \text { a } 9 \text { a } 11 \text {, } \\
& d 3 d 6 d 4 d 10 k 3 k 6 k 4 k 10 \text { f } 1 \text { f2 f } 7 \text { f } 12 \text { p } 1 \text { p2 p } 7 \text { p12 a } 3 a 6 \text { a } 4 \text { a10. }
\end{aligned}
$$

A splitting for the first of these gives the complete 10-arc:

$$
d 3 d 6 k 4 k 10 f 8 f 15 p 9 p 11 a 5 a 16 .
$$


(21) Derived dual semifield plane (BBS4 2-rank 114)

Using [9] (but see Remark 1), 20-sets with 4-nucleus and splitting into complete 10-arcs were found by computation. A 20-set with 4-nucleus 53:

$$
\{1,9,14,17,19,22,25,204,207,212,215,235,240,243,248,257,258,259,263,269\} \text {, }
$$

and a complete 10-arc:

$$
\{1,14,22,25,212,215,235,243,257,258\} \text {. }
$$

\section{(22) Dual derived dual semifield plane (BBS4* 2-rank 114)}

This is one of the planes without hyperovals. Using [9] (but see Remark 1), 20-sets with 4-nucleus and splitting into complete 10 -arcs were found by computation. A 20-set with 4-nucleus 273:

$$
\{6,10,12,15,36,41,46,47,209,210,211,212,241,246,247,248,258,259,260,261\},
$$

and a complete 10-arc:

$\{12,15,36,46,209,210,246,247,258,259\}$.

\section{Acknowledgement:}

The first author acknowledges support of NSF grant GER-9450080. Both authors would like to thank the Department of Computer Science and Engineering and the Center for Communication and Information Science (CCIS) at the University of Nebraska for their hospitality, and the first author would like to thank the Dipartimento di Matematica at the Universitá di Roma 'La Sapienza' for their hospitality, and the C.N.R. (Italy) for financial support.

\section{REFERENCES}

[1] E. F. Assmus, Jr. and J. D. Key. Designs and their Codes. Cambridge University Press, 1992. Cambridge Tracts in Mathematics, Vol. 103 (Second printing with corrections, 1993).

[2] W. Bosma and J. Cannon. Handbook of Magma Functions. Department of Mathematics, University of Sydney, November 1994.

[3] J. W. P. Hirschfeld. Projective Geometries over Finite Fields. Oxford University Press, 1979. 
[4] J. D. Key and M. J. de Resmini. Codewords for a projective plane from sets of type $(s, t)$. European J. Combin., 15:259-268, 1994.

[5] G. Korchmáros and F. Mazzocca. On $(q+t)$-arcs of type $(0,2, t)$ in a desarguesian plane of order q. Math. Proc. Cambridge Philos. Soc., 108:445-459, 1990.

[6] L. Lunelli and M. Sce. k-archi completi nei piani proiettivi desarguesiani di rango 8 e 16. Technical report, Centro Calcoli Numerici, Politecnico di Milano, 1958.

[7] G. Menichetti. $q$-archi completi nei piani di Hall di ordine $q=2^{k}$. Lincei - Rend. Sc. fis. mat. e nat., 56:518-525, 1974.

[8] G. Migliori. Insiemi di tipo $(0,2, q / 2)$ in un piano proiettivo e sistemi di terne di Steiner. Rend. Mat. Appl., 7:77-82, 1987.

[9] T. Penttila, G. F. Royle, and M. K. Simpson. ftp site: cs.uwa.edu.au. Directory: pub/graphs/planes16.

[10] T. Penttila, G. F. Royle, and M. K. Simpson. Hyperovals in the known projective planes of order 16. J. Combin. Des., 4:59-65, 1996.

[11] M. J. de Resmini. On the semifield plane of order 16 with kern GF(2). Ars Combin., 25B:75-92, 1988.

[12] M. J. de Resmini. On the Dempwolff plane. Contemp. Math., 111:47-64, 1990.

[13] M. J. de Resmini. On the derived semifield plane of order 16. Ars Combin., 29B:97-109, 1990.

[14] M. J. de Resmini. On the Johnson-Walker plane. Simon Stevin, 64:113-139, 1990.

[15] M. J. de Resmini. Some remarks on the Hall planes of order 16. Congr. Numer., 70:17-27, 1990 .

[16] M. J. de Resmini. On an exceptional semi-translation plane. In J. W. P. Hirschfeld et al., editor, Finite Geometries and Designs, pages 141-162. Oxford: Oxford University Press, 1991.

[17] M. J. de Resmini. On the semifield plane of order 16 with kern GF(4). In A. Barlotti et al., editor, Combinatorics '88, Vol. 2, pages 369-390. Mediterranean Press, 1991. Research and Lecture Notes in Mathematics.

[18] M. J. de Resmini. On the classical semitranslation plane of order 16. Ars Combin., 46:191-209, 1997.

[19] M. J. de Resmini. On the Mathon plane. J. Geom., 60:47-64, 1997.

[20] M. J. de Resmini and L. Puccio. Some combinatorial properties of the dual Lorimer plane. Ars Combin., 24A:131-148, 1987. 
J. D. Key

Department of Mathematical Sciences Clemson University

Clemson SC 29634

U.S.A

Eingegangen am 1. November 1996
M. J. de Resmini

Dipartimento di Matematica

Universitá di Roma 'La Sapienza'

I-00185 Rome

Italy 